\title{
Modeling Dopamine and Serotonin Systems in a Visual Recognition Network
}

\author{
Stephen Paslaski, Courtland VanDam, and Juyang Weng Fellow, IEEE
}

\begin{abstract}
Many studies have been performed to train a classification network using supervised learning. In order to enable a recognition network to learn autonomously or to later improve its recognition performance through simpler confirmation or rejection, it is desirable to model networks that have an intrinsic motivation system. Although reinforcement learning has been extensively studied, much of the existing models are symbolic whose internal nodes have preset meanings from a set of handpicked symbolic set that is specific for a given task or domain. Neural networks have been used to automatically generate internal (distributed) representations. However, modeling a neuromorphic motivational system for neural networks is still a great challenge. By neuromorphic, we mean that the motivational system for a neural network must be also a neural network, using a standard type of neuronal computation and neuronal learning. This work proposes a neuromorphic motivational system, which includes two subsystems - the serotonin system and the dopamine system. The former signals a large class of stimuli that are intrinsically aversive (e.g., stress or pain). The latter signals a large class of stimuli that are intrinsically appetitive (e.g., sweet and pleasure). We experimented with this motivational system for visual recognition settings to investigate how such a system can learn through interactions with a teacher, who does not give answers, but only punishments and rewards.
\end{abstract}

\section{INTRODUCTION}

Alan Turing indirectly proposed the possibility of a developmental agent, he called a "child machine" [26]. The child machine is an artificial agent that learns its goals and how to accomplish these goals through interacting with the environment, much like a human child. We are not born with goals. We have to learn from our environment what our goals are, and we have to figure out how to accomplish these goals. Humans learn through a developmental cycle. Babies are born and begin interacting with the environment in order to learn their goals.

Some have proposed agents that do not learn through a developmental cycle. The programmer gives the agent a taskspecific goal and the agent completes the task. According to Searle's Chinese room argument, an agent is not considered intelligent if it only processes symbols [20]. An agent that can only process symbols is limited in the number of goals it can understand and achieve. Humans can understand and achieve a variety of goals. If an artificial agent develops through a cycle then it may be able to accomplish muddier tasks that are easy for a human and currently hard for a computer.

Jacob Brown, James Daly, and Juyang Weng are with Michigan State University, East Lansing, MI, USA (email \{paslask1, vandamco, weng\}@cse.msu.edu). Juyang Weng is also with the MSU Cognitive Science Program and the MSU Neuroscience Program. The authors would like to thank Majtoba Solgi for his aid in providing the facial images used for both testing and training.
Weng et al. developed a theory about how Turing's child machine could exist, called autonomous mental development (AMD) [31]. AMD tries to model the development of a human baby within a machine. The development consists of three stages: prenatal, birth, and development. During the prenatal stage, the agent receives its developmental program and some states are initialized. For a human baby, these initial states are its reflexes and some motor control necessary for survival. At birth, the developmental program begins to run. The agent interacts with its environment and uses its developmental program to help it learn new tasks during its developmental stage.

One major portion of learning is distinguishing good behavior from bad. An agent does this based on the rewards and punishments it receives. Learning to distinguish good from bad helps with survival. If a baby learns that putting its hand into the fire hurts, it does not want to do that again. In the human brain, the pain is learned from the release of serotonin in the brain and the rewards come from dopamine. The roles of serotonin and dopamine fall into the category of neuromodulation.

A major advantage of DN with neuromodulation over other supervised learning (e.g., action supervised) techniques is the capability for autonomous learning. The punishments and rewards from the physical world (from the simulated environment here) potentially enable a robot to learn on its own without requiring a human teacher to tediously provide the exactly correct action at every time frame. That is, the environment gives easier "bad" or "good" information, instead of the correct action itself. We do not deal with the time delay problem [21] in this work, requiring the punishment and reward to be timely correct (e.g., a touch on a hot pot gives immediately pain).

Our goal is to model the developmental brain, which learns from pain (serotonin) and sweet (dopamine) signals to the brain. In section 2, we discuss related work. Section 3 is our theory. Section 4 is our algorithm. We present our results in section 5 and conclude in section 6 .

\section{RELATED WORK}

We discuss related work in this section.

\section{A. Symbolic value systems}

Sutton \& Barto 1981 [25] modeled rewards as positive values that the system learns to predict. Ogmen's work [16] was based on Adaptive Resonance Theory (ART), which took into account not only punishments and rewards, but also the 
novelty in expected punishments and rewards, where punishments, rewards, and novelty are all based on a single value. Kakade \& Dayan [10] proposed a dopamine model, which uses novelty and shaping to drive exploration in reinforcement learning, although they did not provide source of information for novelty nor a computational model to measure the novelty. Oudeyer et al. 2007 [17] proposed that the objective functions for a robot uses as a criterion to choose an action fall into three categories, (1) error maximization, (2) progress maximization, and (3) similarity-based progress maximization. Huang \& Weng 2007 [9] proposed an intrinsic motivation system that prioritizes three types of information with decreasing urgency: (1) punishment, (2) reward, and (3) novelty. As punishment and rewards are typically sparse in time, novelty can provide temporally dense motivation even during early life. Krichmar 2008 [11] provided a survey that includes five types of neural transmitters. Singh et al, 2010 [21] adopted an evolutionary perspective and define a new reward framework that captures evolutionary success across environments.

\section{B. Psychological and biological studies}

Psychological studies have provided rich evidence about the existence of the motivational system [13], [14], [22], [23], [19]. It is known that the motivational system is important to the autonomous learning of the brain.

The effects of dopamine and serotonin are not completely understood, according to the literature. Both serotonin and dopamine come in many different forms. Some forms of dopamine are related to reward and some serotonin is related to aversion and punishment. There are other forms of serotonin and dopamine that have different effects that are not widely understood [10], [3]. We will focus on the forms of dopamine and serotonin that affect the brain as rewards and punishment respectively.

Dopamine is associated with reward prediction [10]. If an agent gets a reward, then dopamine is released in the brain. If an agent is expecting a reward, dopamine is also released. For example, if a dog is trained that it always gets food when it hears a bell, then dopamine will be released into the brain at the time that it normally would receive the food. If the dog does not get food at the expected time, then the dopamine levels will drop below normal [10] for a short period of time.

Serotonin is dopamine's counterpart [5]. Dopamine excites the neurons while serotonin inhibits them. One specific type of serotonin with this effect is 5HT. Serotonin leads to behavior inhibition and aversion to punishment [5]. For example, if a monkey pushes a lever and receives a shock, then it will avoid pressing that lever [10]. There are two parts of the brain that release serotonin, the dorsal raphe and the median raphe [5]. The dorsal raphe connects serotonin to all of the areas that have dopamine connections [5], [11]. Serotonin from the dorsal raphe interacts with dopamine to cause the agent to avoid behaviors that the dopamine encourages [5].

\section{Neuromorphic value systems}

Almassy et al. 1998 [1], further refined in Sporns et al. 2000 [24], proposed a neuromorphic architecture for learning primary and seconding conditioning that tend to avoid actions that lead to punishments and adopt actions that lead to reward. Cox \& Krichmar 2007 [4] experimented with a neuromorphic architecture that integrates three types of neurotransmitters, 5-HT, DA and Ach with Ach for increased attention efforts.

In a neuromorphic system, how the neuromorphic value system interacts with the neuromorphic sensorimotor system is also unknown. The Darwin work [1], [24] uses appetitive and aversive stimuli to directly link the corresponding appetitive and aversive behaviors, respectively. Many symbolic methods associate each symbolic long-term behavior with a value, so that a value-based selection mechanism arbitrates which symbolic long-term behavior is executed [27]. Therefore, the value system is like an approval system. Such an approval idea ran into problems with neuromorphic systems. For example, Merrick 2011 [15] proposed an network architecture in which the value system acts like an approval system for sensory inputs, like symbolic systems. Thus, her architecture requires that each neuron in the motivation layer to pass the synaptic weights (not a single response value) to the succeeding neurons. There seems no evidence yet that a neuron can transmit its synaptic weights. Therefore, we do not classify Merrick's model as neuromorphic.

\section{Objectives of our neuromorphic motivational system}

Our motivated neuromorphic architecture is based on a recent network called Developmental Network (DN), which deals with both space and time in an integrated way - temporal context is recursively "folded" into the spatial area like a finite automaton (FA) so that current motor state (response pattern) represents all the temporal context attended at the current time and they are all treated equivalent. Furthermore, all future processing is based such an equivalence. An automata model, called finite automata, along with its properties can clearly explain the principle of DN [29] in dealing with time.

Base on the DN framework, we propose a new neuromorphic architecture with an neuromorphic intrinsic value system. There is no need to directly link an aversive stimuli directly to an avoidance behavior, since our primitive actions are all short (single-frame time), shared by aversive and appetitive behaviors. The architecture does not require synaptic weights to be transmitted to succeeding neurons. In the new architecture, the neuromorphic motivation system develops in parallel with the basic neuromorphic sensorimotor system. The architecture enables the two system to interact in the motor area, via a simple collateral triplets, which is supposed to be hardwired for each primary motor neuron. In this way, each primary motor neuron can be excited by an appetitive stimulus, inhibited by an aversive stimulus, or both currently. 

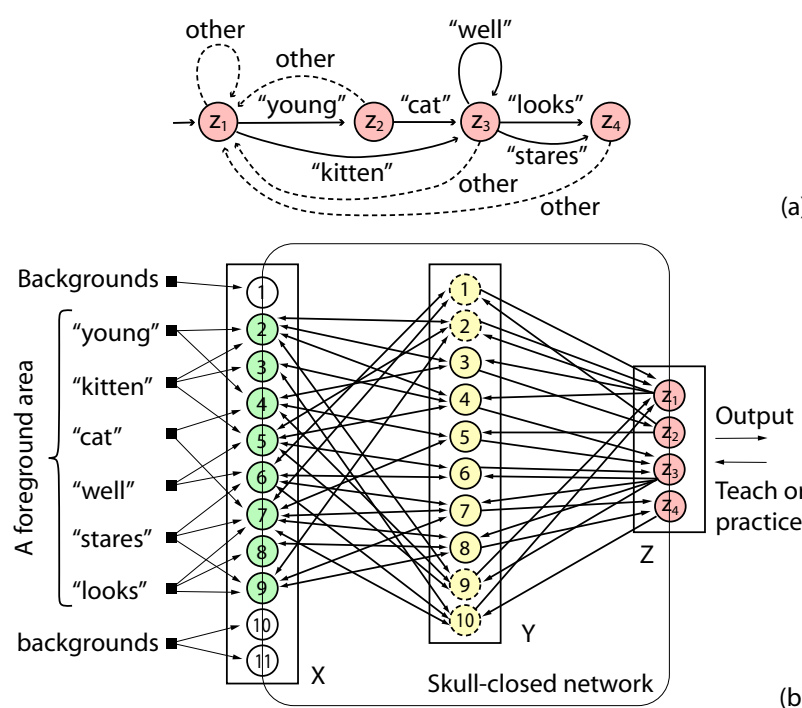

(a)

(b)

Fig. 1. Conceptual correspondence between an Finite Automaton (FA) with the corresponding DN. (a) An FA, handcrafted and static. (b) A corresponding DN that simulates the FA. It was taught to produce the same input-out relations as the FA in (a). The set of symbols $\Sigma=\left\{\sigma_{i} \mid i=1,2, \ldots, l\right\}$ corresponds to the $l$ canonical vectors in the $l$-dimensional vector space $X$. The set of state symbols $Q=\left\{z_{1}, z_{2}, \ldots, z_{m}\right\}$ corresponds to the $m$ canonical vectors in the $m$-dimensional vector space $Z$. In general, however, the dimensions of $X$ and $Z$ are much smaller, by using non-canonical vectors as $X$ in (b). (c) A comparison of the operational mode of the FA and the DN. Pink areas are human designed or human taught. Yellow areas are autonomously generated (emergent and developed).

\section{THEORY}

Humans go through three stages in developing their brain: the prenatal stage, birth, and the development stage. During the prenatal stage, the human brain creates neurons and connects them according to the genome. In our agent, we do not have a genome. We create the developmental program and the layers for the neurons during the prenatal stage and we connect the layers during initialization. At birth, the brain state is initialized. A baby takes its first breath and starts interacting with its environment. For a human, all of the neurons exist at birth, the brain does not create more neurons during the developmental stage [18], [2], [7]. To model human development, we initialize all of the neurons in each layer at birth and begin the developmental program in our agent.

During the developmental stage, the agent interacts with its environment and tries to determine the most appropriate action. The agent uses its sensors to observe the environment and actuators to change the environment. In our program, the sensors observe face images and the actuators attempt to determine to which person (class) the image belongs. For an intelligent agent, it would use its observations to determine its goal during this stage. Initially the agent does not know that its goal is to guess the class of each image. It learns that this is its goal during the developmental stage.

\section{A. Developmental networks}

Developmental Network is the basis of a series of WhereWhat Networks, whose 4th version WWN-4 appeared in [12]. A simplest version of a Developmental Network (DN) has three areas, the sensory area $X$, the internal area $Y$ and the motor area $Z$, with an example in Fig. 1(b). The internal neurons in $Y$ have connection with both the sensory end $X$ and the motor end $Z$.

The largest scale account of neural anatomy so far seems the work of visual and motor systems by Felleman \& Van Essen [8], which reported that the brain is not a cascade of areas, but a network of areas. Every brain area connects not only to the neighboring areas but also directly to many other areas, including the very early LGN and the very late motor cortex. All connections are almost always bi-directional. The DN model hypothesizes that all such connections are mainly due to the statistics of firing activities, prenatally and postnatally. If the DN models the brain, $Y$ contains all the brain internal areas. A DN can also model a particular area of the brain: $Y$ is the brain area, and all its connected areas are $X$ and $Z$, with $X$ closer to sensors and $Z$ closer to motors. The $Y$ area contains multiple types of neurons. We use a separate neuronal map for each type, although typically different types of neurons reside in the same physical space in the brain. Shown in Fig. 2, one map (green) represents dense regular neurons for basic signal processing. Other two maps (blue and pink) represent sparse dopaminergic and serotoninergic neurons, respectively, for neuromodulation.

The DP for DNs is not task-specific as suggested for the brain in [31] (e.g., not concept-specific) at the birth time. In contrast to a static FA, the motor area $Z$ of a DN can be directly observed by the environment (e.g., by the teacher) and thus can be calibrated through interactive teaching from the environment. The environmental concepts are learned incrementally through interactions with the environments. For example, the "young" object makes the pixels 2 and 4 bright and all other green pixels dark. However, such an image from the "young" object" is not known during the programming time for the DP. Compared with symbolic reinforcement learning [21], the DN with neuromodulation here does not assume a particular task (e.g., no hand-picked set of taskspecific symbols), so in principle a DN is able to learn an open array of tasks that the DP programmer is not aware of during the programming time.

In principle, the $X$ area can model any sensory modality (e.g., vision, audition and touch). The motor area $Z$ serves as both input and output ports. When the environment supervises $Z, Z$ is the input to the network. Otherwise, $Z$ gives an output vector to drive effectors (muscles) to act on the real world. The order from low to high is: $X, Y, Z$. The developmental learning of $\mathrm{DN}$, regulated by its $\mathrm{DP}$, is desirably very mechanical:

\section{Algorithm of $\mathrm{DN}$ :}

1) At time $t=0$, for each area $A$ in $\{X, Y, Z\}$, initialize its adaptive part $N=(V, G)$ and the response vector $\mathbf{r}$, where $V$ contains all the synaptic weight vectors and $G$ 
stores all the neuronal ages.

2) At time $t=1,2, \ldots$, for each area $A$ in $\{X, Y, Z\}$, do the following two steps repeatedly forever:

a) Every area $A$ computes using area function $f$.

$$
\left(\mathbf{r}^{\prime}, N^{\prime}\right)=f(\mathbf{b}, \mathbf{t}, N)
$$

where $f$ is the unified area function described in section Methods; $\mathbf{b}$ and $\mathbf{t}$ are area's bottom-up and top-down inputs, respectively; and $\mathbf{r}^{\prime}$ is its response vector.

b) For each area $A$ in $\{X, Y, Z\}, A$ replaces: $N \leftarrow$ $N^{\prime}$ and $\mathbf{r} \leftarrow \mathbf{r}^{\prime}$.

If $X$ is a sensory area, $\mathbf{x} \in X$ is always supervised and then it does not need any synaptic vector. The $\mathbf{z} \in Z$ is supervised only when the teacher chooses to. Otherwise, $\mathbf{z}$ gives motor output.

Next, we describe the area function $f$. Each neuron in area $A$ has a weight vector $\mathbf{v}=\left(\mathbf{v}_{b}, \mathbf{v}_{t}\right)$. Its pre-action potential is the sum of two normalized inner products:

$$
r\left(\mathbf{v}_{b}, \mathbf{b}, \mathbf{v}_{t}, \mathbf{t}\right)=\frac{\mathbf{v}_{b}}{\left\|\mathbf{v}_{b}\right\|} \cdot \frac{\mathbf{b}}{\|\mathbf{b}\|}+\frac{\mathbf{v}_{t}}{\left\|\mathbf{v}_{t}\right\|} \cdot \frac{\mathbf{t}}{\|\mathbf{t}\|}=\dot{\mathbf{v}} \cdot \dot{\mathbf{p}}
$$

which measures the degree of match between the directions of $\dot{\mathbf{v}}=\left(\mathbf{v}_{b} /\left\|\mathbf{v}_{b}\right\|, \mathbf{v}_{t} /\left\|\mathbf{v}_{t}\right\|\right)$ and $\dot{\mathbf{p}}=(\dot{\mathbf{b}}, \dot{\mathbf{t}})=(\mathbf{b} /\|\mathbf{b}\|, \mathbf{t} /\|\mathbf{t}\|)$.

To simulate lateral inhibitions (winner-take-all) within each area $A$, top $k$ winners fire. Considering $k=1$, the winner neuron $j$ is identified by:

$$
j=\arg \max _{1 \leq i \leq c} r\left(\mathbf{v}_{b i}, \mathbf{b}, \mathbf{v}_{t i}, \mathbf{t}\right) .
$$

The area dynamically scale top- $\mathrm{k}$ winners so that the top- $\mathrm{k}$ responses with values in $[0,1]$. For $k=1$, only the single winner fires with response value $y_{j}=1$ and all other neurons in $A$ do not fire. The response value $y_{j}$ approximates the probability for $\dot{\mathbf{p}}$ to fall into the Voronoi region of its $\dot{\mathbf{v}}_{j}$ where the "nearness" is $r\left(\mathbf{v}_{b}, \mathbf{b}, \mathbf{v}_{t}, \mathbf{t}\right)$. We will see below that for symbol-equivalent vectors, the top-1 match is always perfect: $\dot{\mathbf{v}}_{j}=\dot{\mathbf{p}}$.

All the connections in a DN are learned incrementally based on Hebbian learning - cofiring of the pre-synaptic activity $\dot{\mathbf{p}}$ and the post-synaptic activity $y$ of the firing neuron.

It has been proved that the general-purpose DP can incrementally grows a generative DN to simulate any given finite automaton (FA)on the fly, [28], provided that the $Z$ area of the DN is supervised when the DN observes each new state transition from the FA. This indicates that our DN is not like other neural networks that can do pattern recognition, but not effective in logic reasoning (e.g., generalization for sequences not observed).

\section{B. Serotonin and dopamine systems}

Fig. 2 presents the architecture of DN after it is augmented with a motivational system.

The architecture links all pain receptor with raphe nuclei (RN) located in the brain stem - represented as an area, which has the same number of neurons as the number of pain sensors. Every neuron in RN releases serotonin.

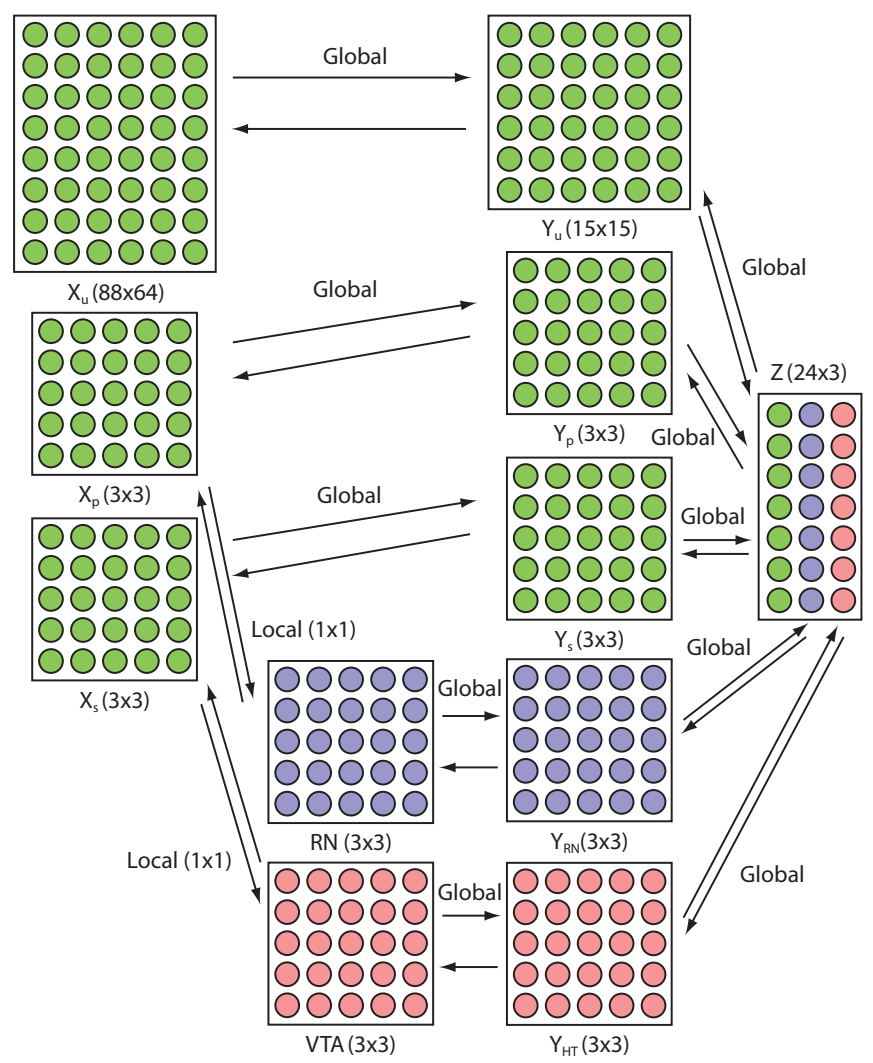

Fig. 2. A DN with a motivational system represented by 5-HT and DA. The blue color denotes serotoninergic neurons. The pink color denotes dopaminergic neurons. In the motor area, each row of neurons corresponds to a collateral triplet.

Similarly, the architecture also links all sweet receptors with hypothalamus (HT) - represented as an area, which as the same number of neurons as the number of sweet receptors. Every neuron in the VTA releases dopamine.

For simplicity, we assume that each row in the motor area represents a different action, although this restriction can be relaxed if we allow multiple muscles to fire together to execute composite actions.

Within such a motivated neural network, the motor area is denoted as $Z=\left(\mathbf{z}_{1}, \mathbf{z}_{2}, \ldots, \mathbf{z}_{m}\right)$, where $m$ is the number of muxels. Each $\mathbf{z}_{i}$ has three neurons $\mathbf{z}_{i}=\left(z_{i u}, z_{i p}, z_{i s}\right)$, where $z_{i u}, z_{i p}, z_{i s}$ are unbiased, pain and sweet, respectively, $i=$ $1,2, \ldots, m . z_{i p}$ and $z_{i s}$ are serotonin and dopamine collaterals, associated with $z_{i u}$, as illustrated by the $Z$ area in Fig. 2 .

Whether the action $i$ is released depends on not only the response of $z_{i u}$ but also those of $z_{i p}$ and $z_{i s} . z_{i p}$ and $z_{i s}$ report how much negative value and positive value are associated with the $i$-th action. They form a triplet for each action $\mathbf{z}_{i}$. We user the following rule for each motor triplelet:

Each motivated action is a vector $\mathbf{z}_{i}=\left(z_{i u}, z_{i p}, z_{i s}\right)$ in $Z=$ $\left(\mathbf{z}_{1}, \mathbf{z}_{2}, \ldots, \mathbf{z}_{m}\right), i=1,2, \ldots, m$. The response of the action neuron is determined by

$$
z_{i u} \leftarrow z_{i u}\left(1+z_{i s}-\alpha z_{i p}\right)
$$

with a very large constant $\alpha$. If $z_{i u}<0, z_{i u} \leftarrow 0$, since 
the response value is never zero. In other words, if $z_{i p}>0$, the corresponding action neuron mostly likely does not fire, as pain is the most dominant factor to avoid. Otherwise, $z_{i s}$ boosts the pre-action potential for the $i$-th action to be released.

Then the $j$-th action is released where

$$
j=\arg \max _{1 \leq i \leq m}\left\{z_{i u}\right\} .
$$

That is, the primitive action released at this time frame is the one that has the highest value after excitation and inhibition with every collateral triplets.

\section{Algorithm}

The developmental stage can be broken down into four ages for each input. In the first stage, the agent simply observes the image and does nothing. This makes sense for babies because they do not have control over most of their muscles. They spend a lot of time watching their environment before they are able to do anything. For the second age, the baby needs to learn the difference between good and bad. In babies, the genome developed the brain to understand the difference between pain and sweet. If the baby is in pain, the brain releases serotonin to discourage an action. If the baby expects a reward, the brain releases dopamine. In our program, the agent does not have dopamine and serotonin stored in the brain, so we use the second age to teach the agent the difference between pain and sweet. At this stage, the agent learns that its goal is to reduce the amount of serotonin it receives and increase the amount of dopamine it receives. In the third age, the baby starts interacting with its environment. The baby moves around and either gets rewards, like its mom talking sweetly to it, or punishment, such as pain. In our program, we give the agent a picture and ask for which class this image belongs to. If the agent guesses it right, we give it a reward with artificial dopamine. If it guesses wrong, we punish the agent with artificial serotonin. Through trial and error, our agent will keep guessing until it gets the image right. Babies will keep trying different actions until they find the ones that get them the reward, just as our agent keeps trying until it gets the reward. For the last age, we test our agent's accuracy. We give it each image and test whether it can guess the class correctly without giving it feedback regarding whether or not it guessed right.

Our algorithm is based on Lobe Component Analysis (LCA) developed by [30]. LCA is loosely based on Principal Component Analysis (PCA). In PCA, the goal is to find the principal components that are most the descriptive features of the dataset. Our dataset is a series of face images, each one with a label of who the face belongs to. In LCA, we are looking for lobe components [30]. The brain in our program has three layers, defined by LCA. The top layer, the sensory layer X, is the images that we feed into the program. The middle layer, Y, is the brain state. The bottom layer, $\mathrm{Z}$, consists of the neurons that control the motor output. This layer consists of a neuron for each action, or class. The middle layer uses the images and the class from the top layer and bottom layer respectively to update the brain state in the $\mathrm{Y}$ layer. To determine the size of each of the layers was rather straightforward. For X and $\mathrm{Z}$ vectors it was necessarily the size of the input and output vectors, respectively. The Y layer was less restricted and after trying several different sizes we found that having around ten times the number of output classes gave us the best results as it is sufficent to capture intra-class variations but not overfit.

Once LCA begins, the neurons are initialized to each observation [30]. Each observation is normalized to unit length before initializing a neuron. After all of the neurons are initialized, the training stage begins, and the program begins cycling through the images and updating the neurons. The response vectors are used to determine which neurons should fire and update [30]. In the $\mathrm{Z}$ area, a response vector using the best neuron from the $\mathrm{Y}$ area is used to update the $\mathrm{Z}$ area. During the testing stage, the agent uses an observation from its environment and the previous response to find the response of $\mathrm{Y}$. The response vector from the $\mathrm{Z}$ area is used to determine which class the agent should return. The accuracy of the agent is determined by how often the top response from the $\mathrm{Z}$ area matches the class of the observation.

In our program, we expand on LCA. For each layer, we add an area for serotonin and dopamine. In the $X$ layer, this is just 1 bit for each chemical. In the $\mathrm{Y}$ and $\mathrm{Z}$ layers, we divide the layer into three parts; an unbiased part that works like LCA, the sweet part for the effects of dopamine, and the pain part for the effects of serotonin. The dopamine and serotonin adjust which neurons may fire by either exciting the neuron or inhibiting the neuron.

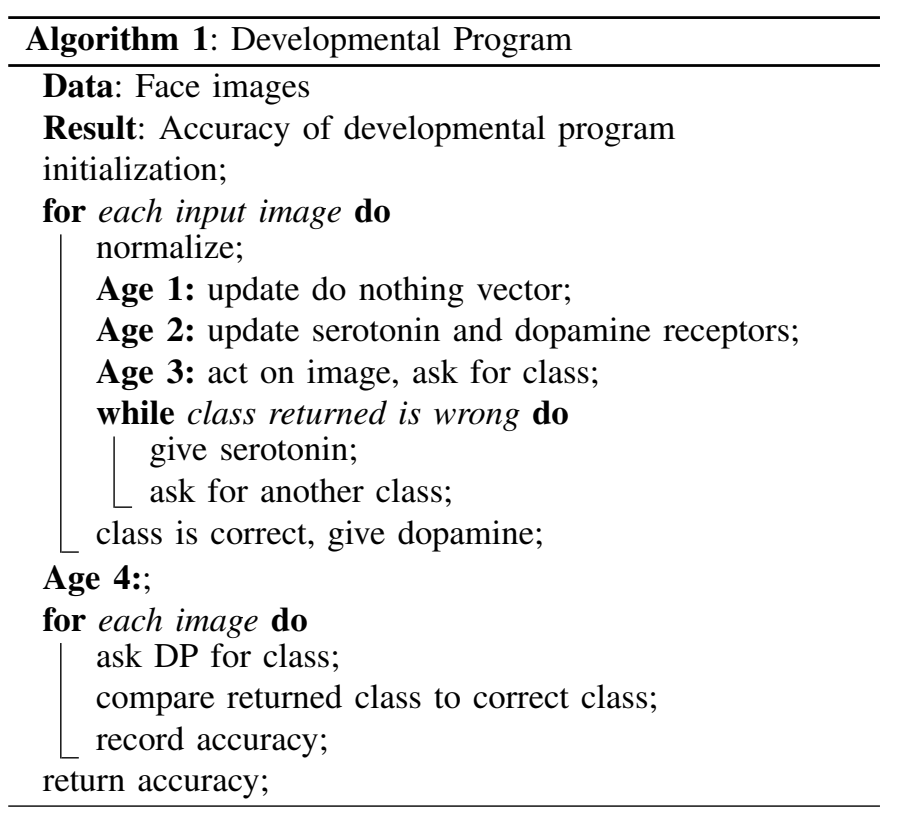

Our program differs from LCA in the number of stages in the program and the number of sub-layers in each layer. In LCA, there are only two stages, one for training and one for testing and the layers are not divided into sublayers. In our agent, the developmental program updates the neurons through four stages, referred to as ages, and each layer has 
three sublayers as described above. In the first age, the agent looks at each image and updates its internal state in the $\mathrm{Y}$ layer. In the $\mathrm{Z}$ area, there is a neuron who's corresponding action is to do nothing. During the first age, the agent only updates this neuron in the $\mathrm{Z}$ area. In the second stage, the agent learns the difference between pain and sweet. For each image, we update the portion of the $\mathrm{Y}$ area that corresponds to serotonin, or pain. Then we update the portion of the $\mathrm{Y}$ area that corresponds to dopamine, or sweet. Ages one and two act similar to the training stage of LCA, but they update different portions of the $\mathrm{Y}$ and $\mathrm{Z}$ areas. In the third age, our program tries to guess the class of the image. On its first guess, it acts similar to the testing stage of LCA. If that guess is wrong, then the next input to the agent is its wrong guess and serotonin. The $\mathrm{Z}$ area then updates its unbiased neuron for this class. After the agent learns that it guessed wrong, it continues to try until it guesses right. Once the agent guesses correctly, the agent receives its correct guess and dopamine. The $\mathrm{Z}$ area for the correct class is updated several times to strengthen that connection. The forth age is very similar to the testing stage of LCA. We give the agent input images and test whether it gives the correct class or not. Once the agent responds to all of the images, we output its accuracy.

\section{EXPERIMENTAL RESULTS}

In the first two ages, as expected, the $\mathrm{Y}$ neurons connected to their neighbors so that neurons with similar weights started to fire together and those with different weights were prevented from firing together. While by far the simplest age, age one where neurons connect and associate with laziness turned out to be one of the most essential stages. As explained in more depth below, too much time in this stage caused connections to be so strong that the tendency to be lazy was very difficult to overcome. Too little time in this stage however caused the phenomenon of neighbors firing together to fail to develop and the neurons were not developed enough to learn in age three.

Age two further strengthened the neural connections and introduced the $\mathrm{Y}$ area to the two neurotransmitters used.

After these first two ages we ended up with strong connections and unbiased $\mathrm{Y}$ neurons that had weights corresponding with the input images that were most likely to cause them to fire.

Due to the fact that initialization of the $\mathrm{Y}$ neurons as well as the sample input images were random, a problem is the very real possibility of a certain class being under-represented in these first two stages which can cause problems in the next age where guesses are made. Similarly, over-representation can cause misclassification in age three where minor feature differences get magnified and also cause inaccurate guesses. The only way to overcome these problems is with an extended age three training period.

One of the key aspects of our experimentation was the correct handling of the neurotransmitters. Since so little is known about the exact biological and physiological impact of both serotonin and dopamine, we were forced to work with little guidance with respect to these. Current research does

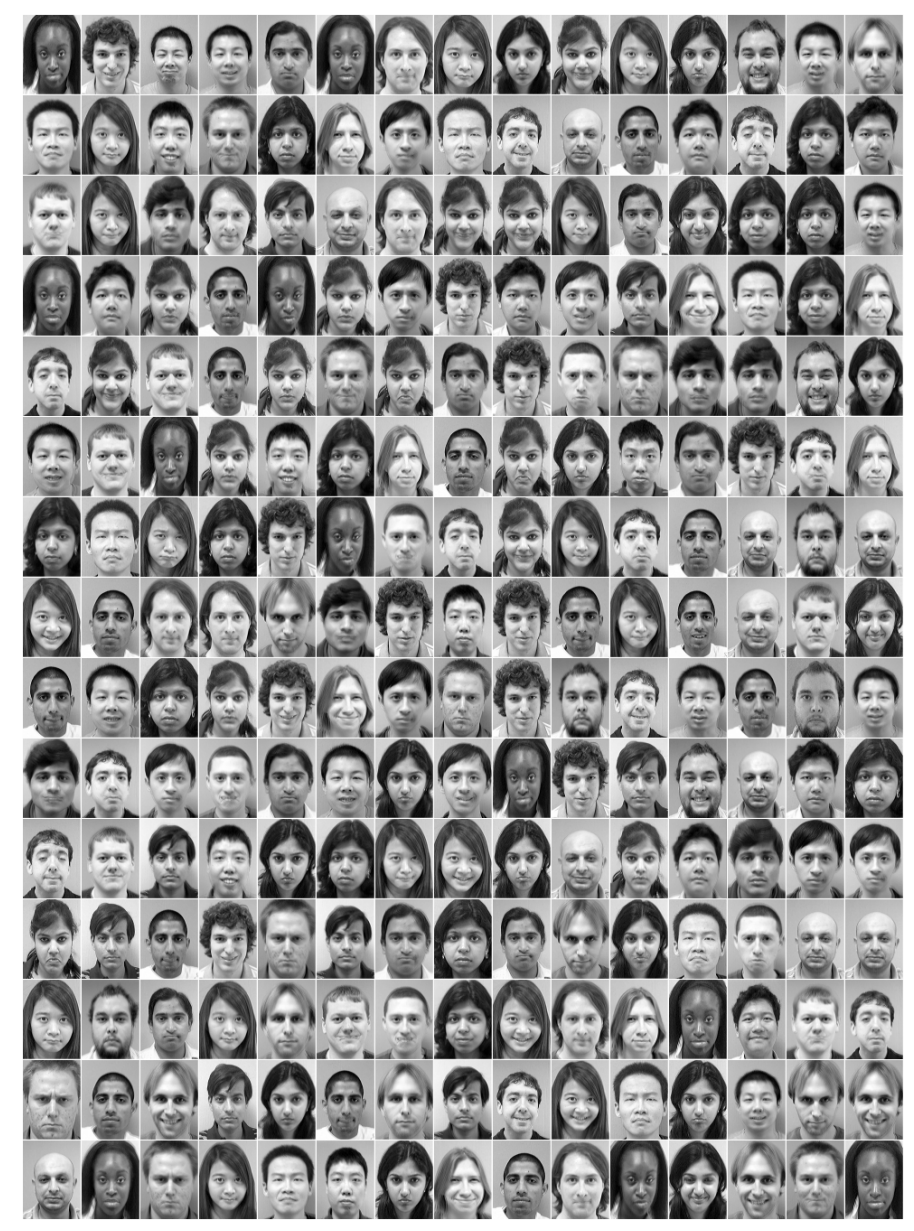

Fig. 3. X weights of the $\mathrm{Y}$ neurons denoting which $\mathrm{Y}$ neurons correspond to which face input When an input face is received in $X$ the top three of these neurons fire and update their weights to simulate Hebbian Learning. The remaining neurons stay unchanged.

seem to indicate a strong initial concentration and a gradual dilution when one or the other is released [3], [6]. Because of this, we decided to send in a full pain or sweet signal of one when punishing or rewarding then let the reduction of the signal be gradual and consistent.

For the serotonin we choose to reduce the pain signal by a small fraction that provided enough time for all other classes to fire before this one fired again. This decision prevented the same few incorrect neurons from taking turns firing and preventing the correct class from ever getting an opportunity to fire, get a reward, and strengthen the association. Even if the correct class turned out to be the worst match at the beginning of age three, it did end up getting the necessary disposition to fire. Towards the beginning of age three there were several misclassifications for each image as there was no predisposition for the correct class to fire; indeed the predisposition was for laziness, which had been promoted, in the first few ages. The behavior of the neurons for the first fifty or so guesses started off as a strategic try-all pattern where the do nothing tendency fired, received pain, then the first class fired, received pain, and continued up until the correct neuron 


\begin{tabular}{|l|r|r|r|r|r|r|r|r|r|r|}
\hline Neurons: & 1 & 2 & 3 & 4 & 5 & 6 & 7 & 8 & 9 & 10 \\
\hline Guess: 1 & 0 & 0 & 0 & 0 & 0 & 0 & 0 & 0 & 0 & 0 \\
\hline Guess: 2 & 0 & 0 & 0 & 0 & 0 & 0 & 0 & 0 & 0 & 0 \\
\hline Guess: 3 & 0.98 & 0 & 0 & 0 & 0 & 0 & 0 & 0 & 0 & 0 \\
\hline Guess: 4 & 0.96 & 0 & 0 & 0 & 0 & 0 & 0 & 0 & 0 & 0 \\
\hline Guess: 5 & 0.94 & 0 & 0 & 0 & 0 & 0 & 0.98 & 0 & 0 & 0 \\
\hline Guess: 6 & 0.91 & 0 & 0 & 0 & 0 & 0 & 0.96 & 0.98 & 0 & 0 \\
\hline Guess: 7 & 0.89 & 0 & 0 & 0 & 0 & 0 & 0.94 & 0.96 & 0 & 0.98 \\
\hline Guess: 8 & 0.87 & 0 & 0 & 0 & 0 & 0 & 0.91 & 0.94 & 0.98 & 0.96 \\
\hline Guess: 9 & 0.85 & 0 & 0 & 0 & 0 & 0 & 0.89 & 0.91 & 0.96 & 0.94 \\
\hline Guess: 10 & 0.83 & 0.98 & 0 & 0 & 0 & 0 & 0.87 & 0.89 & 0.94 & 0.91 \\
\hline Guess: 11 & 0.81 & 0.96 & 0 & 0 & 0 & 0 & 0.85 & 0.87 & 0.91 & 0.89 \\
\hline Guess: 12 & 0.79 & 0.94 & 0 & 0 & 0 & 0 & 0.83 & 0.85 & 0.89 & 0.87 \\
\hline Guess: 13 & 0.77 & 0.91 & 0 & 0 & 0 & 0 & 0.81 & 0.83 & 0.87 & 0.85 \\
\hline Guess: 14 & 0.75 & 0.89 & 0.98 & 0 & 0 & 0 & 0.79 & 0.81 & 0.85 & 0.83 \\
\hline Guess: 15 & 0.73 & 0.87 & 0.96 & 0 & 0 & 0 & 0.77 & 0.79 & 0.83 & 0.81 \\
\hline Guess: 16 & 0.71 & 0.85 & 0.94 & 0 & 0 & 0.98 & 0.75 & 0.77 & 0.81 & 0.79 \\
\hline Guess: 17 & 0.69 & 0.83 & 0.91 & 0 & 0 & 0.96 & 0.73 & 0.75 & 0.79 & 0.77 \\
\hline Guess: 18 & 0.63 & 0.77 & 0.85 & 0 & 0.96 & 0.89 & 0.67 & 0.69 & 0.73 & 0.71 \\
\hline Guess: 19 & 0.61 & 0.75 & 0.83 & 0 & 0.94 & 0.87 & 0.65 & 0.67 & 0.71 & 0.69 \\
\hline Guess: 20 & 0.59 & 0.73 & 0.81 & 0 & 0.91 & 0.85 & 0.63 & 0.65 & 0.69 & 0.67 \\
\hline
\end{tabular}
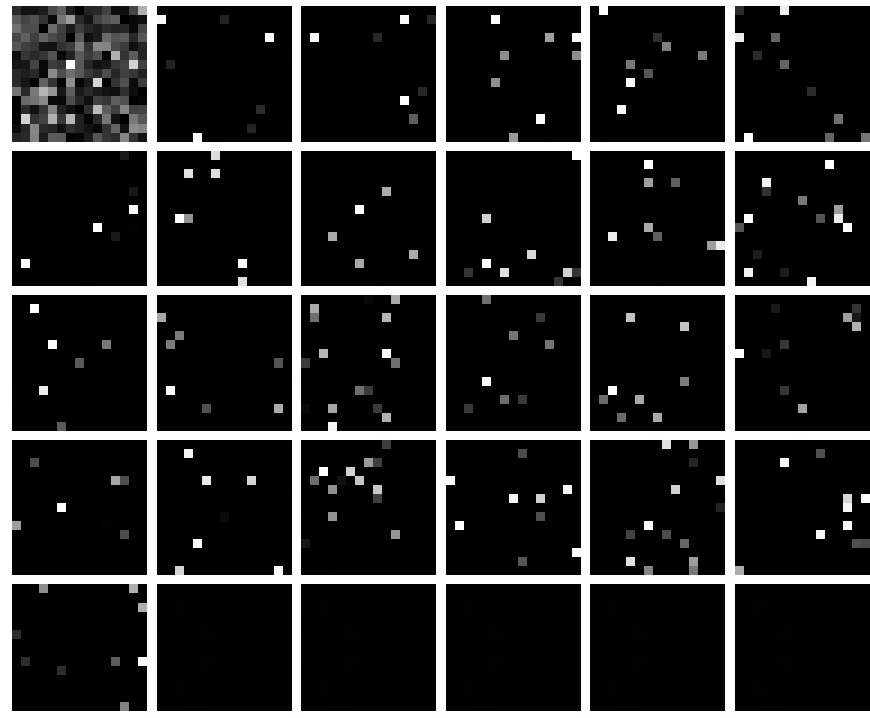

Fig. 5. $\quad \mathrm{Y}$ weights of the $\mathrm{Z}$ neurons signifying which neurons fire for which class. The first square represents the weights of the neuron for the do nothing action. Each of the following neuron weights are those for a specific class and show which Y neurons were most likely to cause them to fire. White represents a high tendency while black represents a low or non-existent tendency. The last five square were unused neurons. beginning of training. The signal starts strong and gradually decreases until there is no more serotonin preventing firing. At this point if the previously suppressed neuron is the best match and can overcome any reward associated with another class, it will have the opportunity to re-fire.

fired and was rewarded. As expected, this behavior became more scarce as the neurons adapted and were able to correctly determine which class they represented.

With dopamine we chose to make the decrease still gradual but faster so that this does not reward the correct class in future guesses.

After several random input images were presented to the $\mathrm{Y}$ neurons to guess, a pattern started to emerge where only a select few Y neurons firing would automatically cause the associated $\mathrm{Z}$ neuron to fire. As this pattern got reinforced with more training, the do nothing neuron was over-powered by the correct neuron and ceased to fire. In general, the neurons that were neither over-represented, having more than fifteen active $\mathrm{Y}$ neurons, nor under-represented, having less than five active neurons were the best guessers. In this case, the active $\mathrm{Z}$ neuron weights were strong enough to produce a strong response and the same $\mathrm{Y}$ neurons tended to fire together which further enhanced the response.

One of the phenomena briefly mentioned above is the potential over-tendency to do nothing if too much time was spent in the first two ages where this was the only available action. Too much time strengthened the $\mathrm{Y}$ neurons and strongly associated them with this lazy action. Even with only a moderate time spent in these ages produced a strong tendency that was very difficult to overcome and resulted in multiple images being stuck in this classification even if plenty of guessing and reinforcement training was pursued. Noticing that the only misclassifications in age four when we tested was the do nothing action which was strengthened in the first two ages but never fired in age three, we included an option to reduce the weights of a neuron if serotonin was present. Due to the lack of conclusive evidence regarding the role of serotonin in some aspects of neuromodulation, we were unable to determine the viability of this function in physiology.

Overall, with a rather modest 500 random training images in age one and 300 random age two images for both sweet and pain, we got rather conclusive and positive results. We tested the classification before any age three training was done and it came as no surprise that background was the unanimous guess. We then retested it after every training cycle with fifty test images using the option to reduce weights as well as without this option and continued to do this until we had trained with a total of 1000 age three images.

Both with the reduction option on and off, training got off to a similar start and we account for the variance to be due to the random aspect of the training input. In both cases our developmental program rapidly converged to a less than ten percent error rate after only 150 images.

After this, the instance that had the reduction option on achieved a perfect recognition and stayed perfect for the duration of training-testing cycles.

The instance with the reduction option disabled was slightly more sporadic but still remained under the five percent error mark. Throughout all of the cycles, all of the testing images were correctly classified at least once and the few misclassifications were all due to the same five classes, which took turns misclassifying. In some instances it seemed that one correct classification for a face resulted in a misclassification of another face in the same class. While each class had perfect classification at some point during the early testing, this was 


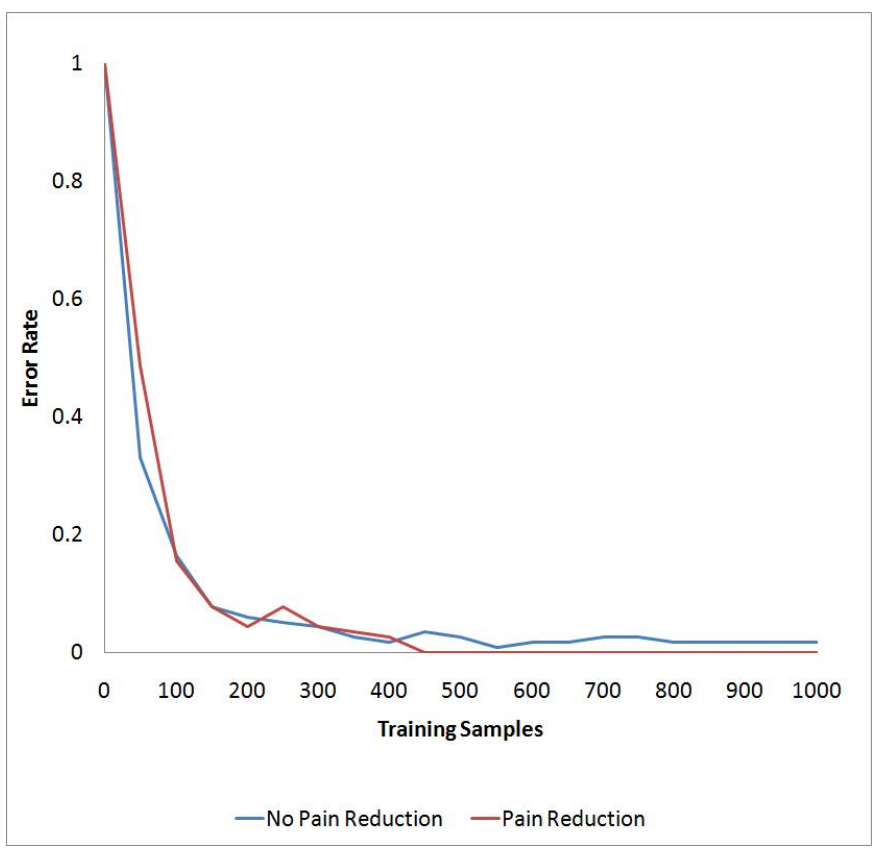

Fig. 6. Error rate in recognition for testing with different training durations and different neural mechanisms.

rather inconsistent for some classes. With enough training and testing however, we do eventually end up with perfect classification. It simply takes much longer with some of the classes that were unfortunate in age one and had their tendency to be lazy strengthened more than was necessary. Eventually enough iterations of supervision strengthens the correct class neuron enough to over-power the do nothing action and thus suppress it. Without the option to reduce weights with the presence of serotonin this convergence simply takes much longer.

\section{CONCLUSIONS AND DISCUSSION}

We proposed a neuromorphic motivational system to be embedded into the Developmental Network. We tested the motivated network for the task of classification of well-framed face images.

By mimicking only two of the neuromodulatory transmitters in the brain we were able to train our program to correctly identify faces with a very high level of confidence and without ever imposing the correct action on the motor neurons. This provides a framework for an emergent system to autonomously learn using punishment and reward.

\section{REFERENCES}

[1] N. Almassy, G. M. Edelman, and O. Sporns. Behavioral constraints in the development of neural properties: A cortical model embedded in a real-world device. Cerebral Cortex, 8(4):346-361, 1998.

[2] S. Carey. Conceptual Change in Childhood. The MIT Press, Cambridge, Massachusetts, 1985.

[3] S. Cavallaro. Genomic analysis of serotonin receptors in learning and memory. Behavioural Brain Research, 195(1):2-6, 2008.

[4] B. Cox and J. Krichmar. Neuromodulation as a robot controller. IEEE Robotics and Automations Magazine, 16(3):72 - 80, 2009.

[5] N. D. Daw, S. Kakade, and P. Dayan. Opponent interactions between serotonin and dopamine. Neural Networks, 15(4-6):603-616, 2002.
[6] V. H. Denenberg, D. S. Kim, and R. D. Palmiter. The role of dopamine in learning, memory, and performance of a water escape task. Behavioural Brain Research, 148(1-2):73-78, 2004.

[7] M. Domjan. The Principles of Learning and Behavior. Brooks/Cole, Belmont, California, fourth edition, 1998.

[8] D. J. Felleman and D. C. Van Essen. Distributed hierarchical processing in the primate cerebral cortex. Cerebral Cortex, 1:1-47, 1991.

[9] X. Huang and J. Weng. Inherent value systems for autonomous mental development. International Journal of Humanoid Robotics, 4(2):407433, 2007.

[10] S. Kakade and P. Dayan. Dopamine: generalization and bonuses. Neural Network, 15:549559, 2002.

[11] J. L. Krichmar. The neuromodulatory system: A framework for survival and adaptive behavior in a challenging world. Adaptive Behavior, 16(6):385-399, 2008.

[12] M. Luciw and J. Weng. Where What Network 3: Developmental top-down attention with multiple meaningful foregrounds. In Proc. IEEE Int'l Joint Conference on Neural Networks, pages 4233-4240, Barcelona, Spain, July 18-23 2010.

[13] A. H. Maslow. A theory of human motivation. Psychological Review, 50(4):370-396, 1943.

[14] A. H. Maslow. Motivation and Personality. Harper and Row, New York, 1 edition, 1954.

[15] K. E. Merrick. A comparative study of value systems for self-motivated exploration and learning by robots. IEEE Trans. Autonomous Mental Development, 2(2):119-131, 2010.

[16] H. Ogmen. A developmental perspective to neural models of intelligence and learning. In D. Levine and R. Elsberry, editors, Optimality in Biological and Articial Networks, page 363395. Lawrence Erlbaum, Hillsdale, NJ, 1997.

[17] P.-Y. Oudeyer, F. Kaplan, and V. Hafner. Intrinsic motivation for autonomous mental development. IEEE Transactions on Evolutionary Computation, 11(2):265286, 2007.

[18] J. Piaget. The Construction of Reality in the Child. Basic Books, New York, 1954.

[19] T. W. Robbins and B. J. Everitt. Neurobehavioural mechanisms of reward and motivation. Current Opinion in Neurobiology, 6(2):228236, April 1996.

[20] J. Searle. Minds, brains, and programs. Behavioral and Brain Sciences, 3(3):417-424, 1980

[21] S. Singh, R. L. Lewis, A. G. Barto, and J. Sorg. Intrinsically motivated reinforcement learning: An evolutionary perspective. IEEE Trans. Autonomous Mental Development, 2(2):70-82, 2010.

[22] R. L. Solomon and J. D. Corbit. An opponent-process theory of motivation: Ii. cigarette addition. Journal of Abnormal Psychology, 81:158-171, 1973.

[23] R. L. Solomon and J. D. Corbit. An opponent-process theory of motivation: I. the temporal dynamics of affect. Psychological Review, 81:119-145, 1974.

[24] O. Sporns, N. Almassy, and G.M. Edelman. Plasticity in value systems and its role in adaptive behavior. Adaptive Behavior, 7(3), 1999.

[25] R.S. Sutton and A.G. Barto. Toward a modern theory of adaptive networks: Expectation and prediction. Psychological Review, 88:135$170,1981$.

[26] A. M. Turing. Computing machinery and intelligence. Mind, 59:433460, October 1950.

[27] C. Watkins and P. Dayan. Q-learning. Machine Learning, 8:279-292, 1992.

[28] J. Weng. A 5-chunk developmental brain-mind network model for multiple events in complex backgrounds. In Proc. Int'l Joint Conf. Neural Networks, pages 1-8, Barcelona, Spain, July 18-23 2010.

[29] J. Weng. A general purpose brain model for developmental robots: The spatial brain for any temporal lengths. In Proc. Workshop on Bio-Inspired Self-Organizing Robotic Systems, IEEE Int'l Conference on Robotics and Automation, pages +1-6, Anchorage, Alaska, May 3-8 2010.

[30] J. Weng and M. Luciw. Dually optimal neuronal layers: Lobe component analysis. IEEE Trans. Autonomous Mental Development, 1(1):68-85, 2009.

[31] J. Weng, J. McClelland, A. Pentland, O. Sporns, I. Stockman, M. Sur, and E. Thelen. Autonomous mental development by robots and animals. Science, 291(5504):599-600, 2001. 